\title{
Efeito de Isolados de Metarhizium anisopliae (Metsch.) Sorok. e Beauveria bassiana (Bals.) Vuill. sobre o Percevejo-do-Colmo do Arroz, Tibraca limbativentris Stal
}

\author{
José F. da S. Martins ${ }^{1}$, Maria G. A. de Lima², Marcos Botton ${ }^{1}$, \\ Jairo J. Carbonari ${ }^{1}$ e Eliane D. Quintela ${ }^{3}$ \\ ${ }^{1}$ Centro de Pesquisa Agropecuária de Clima Temperado/Embrapa, \\ Caixa postal 403, 96001-970, Pelotas, RS. \\ ${ }^{2}$ Empresa Goiana de Pesquisa Agropecuária, Caixa postal 49, 74610-060, Goiânia, GO. \\ ${ }^{3}$ Centro Nacional de Pesquisa de Arroz e Feijão/Embrapa, Caixa postal, \\ 179, 74001-970, Goiânia, GO.
}

An. Soc. Entomol. Brasil 26(2): 277-283 (1997)

Effect of Metarhizium anisopliae (Metsch.) Sorok. and Beauveria bassiana

(Bals.) Vuill. Isolates on Rice Stem Bug, Tibraca limbativentris Stal

\begin{abstract}
The rice stem bug, Tibraca limbativentris Stal (Heteroptera: Pentatomidae), is an important pest of irrigated rice (Oryza sativa) crop in Brazil. The effect of isolates $(\mathrm{CP})$ of the entomopathogenic fungi, Beauveria bassiana (Bals.) Vuill. ( $B b)$ and Metarhizium anisopliae (Metsch.) Sorok. (Ma) was evaluated in the field using cages. In the 1st and 2nd experiments, conidial suspensions at dosages equivalents to $10^{13}$ conidia/ha, were sprayed on the soil and on rice plant stems, where the bugs were concentrated. Significant mortality (M) was caused by isolates CPBb164, CPMa171 and, mainly, by CPMa172 $(46,5 \% \leq M \leq 88,7 \%)$. In the 3rd experiment, conidial sprays and broadcast of fungus on inoculated grain rice at $10^{13}$ conidia/ha were evaluated.. Both applications were effective $(42,7 \% \leq M \leq 70,8 \%)$ in controlling the insect.
\end{abstract}

KEY WORDS: Insecta, Heteroptera, Pentatomidae, Oryza sativa, microbial control.

RESUMO - O percevejo-do-colmo, Tibraca limbativentris Stal (Heteroptera: Pentatomidae), é praga importante da cultura do arroz (Oryza sativa) irrigado no Brasil. O efeito de isolados (CP) dos fungos entomopatogênicos Beauveria bassiana (Bals.) Vuill. (Bb) e Metarhizium anisopliae (Metsch.) Sorok. (Ma), na mortalidade (M) do percevejo, foi avaliado a campo, em três experimentos em gaiolas, incluindo arroz transplantado e infestação artificial com insetos adultos. No $1^{\circ}$ e $2^{\circ}$ experimentos, suspensões equivalentes a $10^{13}$ conídios/ha foram pulverizadas no solo e nos colmos das plantas de arroz, onde os percevejos estavam concentrados. Os isolados CPBb164, CPMa171 e, principalmente, $\mathrm{CPMa} 172$ foram os mais virulentos $(46,5 \% \leq \mathrm{M} \leq 88,7 \%)$. No $3^{\circ}$ experimento, dois métodos de aplicação foram avaliados: pulverização de conídios e distribuição manual de grãos de arroz com material fúngico em desenvolvimento, em dosagens equivalentes a $10^{13}$ conídios/ha. Ambos os métodos de aplicação controlaram eficientemente $(42,7 \% \leq \mathrm{M} \leq 70,8 \%)$ o inseto.

PALAVRAS-CHAVE: Insecta, Heteroptera, Pentatomidae, Oryza sativa, controle microbiológico. 
O percevejo-do-colmo, Tibraca limbativentris Stal (Heteroptera: Pentatomidae), ocorre na maioria das regiões orizícolas do Brasil (Ferreira et al. 1986), principalmente em cultivos irrigados. Danifica plantas de arroz (Oryza sativa) desde o início da fase de perfilhamento, porém, causa maiores prejuízos à produção de grãos, quando perfura os colmos entre a fase de pré-floração e a de formação de panículas (Costa \& Link 1992).

Nos arrozais, o inseto localiza-se na base das plantas de arroz, entre os colmos, preferencialmente onde não há formação de lâmina d'água de irrigação, estando o solo apenas saturado (Botton et al. 1996). Nesses locais, estabelecem-se condições micrometeorológicas de umidade e temperatura propícias ao crescimento da população do inseto (Martins \& Lima 1994) e ao desenvolvimento de fungos entomopatogênicos (Carbonell et al. 1980).

Pesquisas pioneiras, sobre efeito de fungos entomopatogênicos em percevejos que atacam o colmo do arroz, foram realizadas na Ásia, envolvendo, principalmente, o pentatomídeo Scotinophara coarctata (F.) e os fungos Metarhizium anisopliae (Metsch.) Sorok. e Beauveria bassiana (Bals.) Vuill. (Rombach et al. 1986). No Brasil, pesquisas similares com T. limbativentris foram iniciadas com base na premissa de que o hábito de localização do inseto e as condições micrometeorológicas entre os colmos de arroz seriam adequadas ao estabelecimento e disseminação de fungos entomopatogênicos em arrozais (Martins et al. 1986). Estudos em laboratório, simulando condições de campo favoráveis ao crescimento da população de T. limbativentris, permitiram identificar isolados de B. bassiana e M. anisopliae mais virulentos ao inseto (Martins \& Lima 1994). O objetivo desse trabalho foi avaliar o efeito de isolados e de formas de aplicação de ambas as espécies de entomopatógenos sobre $T$. limbativentris, em condições de campo.

\section{Material e Métodos}

Três experimentos, foram realizados na
Embrapa-CPACT, infestando-se plantas de arroz, em gaiolas, com adultos de $T$. limbativentris e aplicando-se isolados $(\mathrm{CP})$ de $M$. anisopliae $(M a)$ e $B$. bassiana $(B b)$. Os isolados (Tabela 1), alguns pré-selecionados quanto à virulência ao inseto (Martins \& Lima 1994), foram obtidos da coleção da EmbrapaCNPAF. O material fúngico foi produzido em sacos de polipropileno, contendo grãos de arroz descascados, autoclavados e mantidos à temperatura de $27 \pm 1^{\circ} \mathrm{C}$, por 15 dias (Marques et al. 1981). A viabilidade dos conídios foi aferida pelo método de germinação, via espalhamento uniforme de 10 microlitros de suspensão de cada isolado em três placas de Petri, com meio de cultura BDA + streptomicina, sendo as placas mantidas em estufa incubadora, a $26^{\circ} \mathrm{C}$, por 48 horas. A viabilidade dos isolados foi superior a $90 \%$, nos três experimentos. Os insetos usados na infestação artificial foram criados em casa de vegetação (Martins \& Lima 1994).

No $1^{\circ}$ experimento (1988), foram avaliados quatro isolados de $M$. anisopliae e um de $B$. bassiana, num delineamento de blocos casualizados, com cinco repetições. A parcela experimental continha 25 touceiras de plantas, da cultivar BR-IRGA 414, eqüidistantes $25 \mathrm{~cm}$. Ao final da fase de perfilhamento, as plantas foram cobertas por gaiolas de tela de náilon $(1,2 \times 1,2 \times 1,2 \mathrm{~m}) \mathrm{e}$ infestadas com 37 insetos (20 machos e 17 fêmeas). A irrigação foi controlada para manter o solo saturado e permitir a localização dos insetos na base das plantas, entre os colmos. As plantas foram pulverizadas com suspensões fúngicas (água destilada $+0,5 \%$ de Tween 80 ), na dosagem de $10^{13}$ conídios/ ha. Na aplicação, com pulverizador manual (BRUDDEN - Modelo P1), foram utilizados $100 \mathrm{ml}$ de suspensão por parcela, atingindo, solo e a base dos colmos, até cerca de $20 \mathrm{~cm}$ de altura. As plantas testemunhas, foram tratadas com solução de $0,5 \%$ de Tween 80 em água destilada. A mortalidade de insetos foi registrada aos 3, 7, 11, 14, 17 e 21 dias após à aplicação dos tratamentos (DAT). Os insetos mortos foram mantidos individualizados em câmara úmida, à $26^{\circ} \mathrm{C}$, até a 
Tabela 1. Isolados (CP) de Beauveria bassiana $(B b)$ e Metarhizium anisopliae $(\mathrm{Ma})$ avaliados quanto à virulência à Tibraca limbativentris, em gaiolas no campo.

\begin{tabular}{lll}
\hline Isolados $^{1}$ & Procedência & Hospedeiro original \\
\hline $\mathrm{CP} B b 20$ & Goiânia & Deois flavopicta (Stal) \\
$\mathrm{CP} B b 164$ & Chapecó-SC & Nezara viridula $(\mathrm{L}$.) \\
$\mathrm{CPMa} 30$ & Goiânia-GO & Deois flavopicta (Stal) \\
$\mathrm{CP} M a 122$ & Goiatuba-GO & Scaptocoris castanea Perty \\
$\mathrm{CPMa} 170$ & Goiânia-GO & Tibraca limbativentris Stal \\
$\mathrm{CPMa} 171$ & Goiânia-GO & Tibraca limbativentris Stal \\
$\mathrm{CPMa} 172$ & Goiânia-GO & Tibraca limbativentris Stal \\
$\mathrm{CPMaE9}$ & Rio N. Sul-ES & Deois flavopicta (Stal) \\
\hline
\end{tabular}

${ }^{1}$ Oriundos da coleção de entomopatógenos da Embrapa-CNPAF.

constatação de crescimento fúngico nos cadáveres.

No $2^{\circ}$ experimento (1989), foram colocados oito casais do inseto/gaiola. Os isolados CPMa30 e CPMaE9 foram substituídos por CPMa170 e CPMa172 e o $\mathrm{CP} B b 20$ por $\mathrm{CP} B b 164$, para pulverizações de $10^{13}$ conídios/ha. No $3^{\circ}$ experimento (1990), foram colocados doze casais/gaiola, sendo comparado o efeito de CPBb164, CPMa 171 e CPMa172, aplicados na dosagem $10^{13}$ conídios/ha, tanto na forma de suspensão de conídios como de grânulos (grãos de arroz utilizados na produção do material fúngico). Os grânulos foram distribuídos manualmente, entre os colmos, próximos à base das plantas ou atingindo a superfície do solo. No $2^{\circ}$ e $3^{\circ}$ experimentos, o número de insetos mortos e infectados foi registrado aos 20 DAT. Os cadáveres não foram retirados das gaiolas, visando manter a fonte de inóculo. Os experimentos foram prolongados, respectivamente, até 90 e 100 DAT, quando as gaiolas foram removidas para contagem de insetos vivos (ninfas de $3^{\circ}, 4^{\circ} 5^{\circ}$ ínstar e adultos).

Para análise de variância (SAS Institute 1985), o número (N) de insetos vivos, mortos e infectados foi transformado em $\mathrm{N}+0,5$, sendo as médias comparadas pelo teste de Tukey $(\mathrm{P} \leq 0,10)$. A eficiência de controle exercida pelos isolados dos fungos foi calculada pela fórmula de Abbott (1925).

\section{Resultados e Discussão}

No $1^{\circ}$ experimento, CPMa 171 foi o mais virulento, causando $46,5 \%$ de mortalidade em T. limbativentris (Tabela 2). O número de insetos mortos com infecção fúngica confirmada (I) foi baixo, em relação a todos os isolados $(0 \leq \mathrm{I} \leq 4,8)$. Somente CPMa 171 diferiu significativamente da testemunha e não ocorreu crescimento fúngico em insetos tratados com CPBb20.

No $2^{\circ}$ experimento, CPMa 172 foi o mais eficiente, provocando até 20 e 90 DAT, reduções de 88,7 e $94,3 \%$ na população de $T$. limbativentris, respectivamente. CPMa171 não confirmou o nível de eficiência demostrado no experimento anterior e causou apenas 39,6\% de mortalidade (Tabela 3). O número de insetos mortos com estruturas fúngicas também foi baixo no $2^{\circ}$ experimento $(1,0 \leq \mathrm{I} \leq 5,4)$.

No $3^{\circ}$ experimento, apesar do número de insetos mortos com infecção confirmada ser novamente baixo $(1,8 \leq \mathrm{I} \leq 5,0)$, os isolados CPBb164, CPMa171 e CPMa172, na forma de suspensão de conídios ou de grânulos, reduziram significativamente a população de T. limbativentris (Tabela 4). A pulverização com suspensão de conídios, contudo, tendeu ser mais eficiente que a aplicação manual dos 
Tabela 2. Mortalidade ${ }^{1}$ e infecção de Tibraca limbativentris até 21 dias após o tratamento ${ }^{2}$ com isolados (CP) de Beauveria bassiana $(\mathrm{Bb})$ e Metarhizium anisopliae (Ma), em gaiolas no campo.

\begin{tabular}{|c|c|c|c|c|c|c|c|c|}
\hline \multirow[t]{2}{*}{ Tratamento } & \multicolumn{6}{|c|}{$\begin{array}{l}\mathrm{N}^{\mathrm{o}} \text { Cumulativo de Insetos Mortos } \\
\text { (Dias pós-tratamento) }^{4}\end{array}$} & \multirow{2}{*}{$\begin{array}{l}\text { Mort. } \\
(\%)^{3}\end{array}$} & \multirow{2}{*}{$\begin{array}{l}\text { Insetos } \\
\text { Infect. } \\
\left(\mathrm{N}^{\mathrm{o}}\right)^{4}\end{array}$} \\
\hline & 3 & 7 & 11 & 14 & 17 & 21 & & \\
\hline Testesm. & $1,0 \mathrm{a}$ & $2,4 \mathrm{a}$ & $3,6 \mathrm{a}$ & $4,4 \mathrm{a}$ & $5,4 \mathrm{a}$ & $5,8 \mathrm{a}$ & - & 0 \\
\hline $\mathrm{CPB} b 20$ & $3,8 \mathrm{ab}$ & $6,0 \mathrm{a}$ & $6,8 \mathrm{a}$ & $9,4 \mathrm{a}$ & $10,4 \mathrm{~b}$ & $10,6 \mathrm{ab}$ & 12,9 & 0 \\
\hline СРМa122 & $4,4 \mathrm{~b}$ & $6,0 \mathrm{a}$ & $7,0 \mathrm{a}$ & $9,0 \mathrm{a}$ & $10,4 \mathrm{~b}$ & $12,8 \mathrm{~b}$ & 18,9 & $0,6 \mathrm{a}$ \\
\hline СРМа30 & $1,4 \mathrm{a}$ & $2,6 \mathrm{a}$ & $3,8 \mathrm{a}$ & $5,8 \mathrm{a}$ & $7,2 \mathrm{ab}$ & $8,0 \mathrm{ab}$ & 5,9 & $0,6 \mathrm{a}$ \\
\hline СРMaЕ9 & $2,8 \mathrm{ab}$ & $4,8 \mathrm{a}$ & $7,2 \mathrm{a}$ & $9,0 \mathrm{a}$ & $11,2 \mathrm{~b}$ & $12,0 \mathrm{~b}$ & 16,8 & $0,2 \mathrm{a}$ \\
\hline СРМa171 & $3,8 \mathrm{ab}$ & $6,0 \mathrm{a}$ & $8,6 \mathrm{a}$ & $5,2 \mathrm{~b}$ & $20,6 \mathrm{c}$ & $23,0 \mathrm{c}$ & 46,5 & $4,8 \mathrm{~b}$ \\
\hline
\end{tabular}

${ }^{1}$ Número inicial de insetos/gaiola $=37$ (20 machos e 17 fêmeas).

${ }^{2}$ Pulverização com suspensão fúngica (dosagem $=10^{13}$ conídios $/$ ha)

${ }^{3}$ Mortalidade cumulativa (\%) $=[($ Mort. do trat. - Mort. da test. $) / 37]$ X 100.

${ }^{4}$ Médias com mesma letra não diferem significativamente (Tukey: $\mathrm{P}<0,10$ ).

grânulos, sendo estabelecida hipótese a respeito: a pulverização, ao distribuir os conídios mais uniformemente, forma mais sítios de contaminação por área tratada e atinge de imediato maior número de insetos, enquanto a contaminação via material fúngico distribuído manualmente somente ocorre após o inseto ter contato com pontos esparsos das

Tabela 3. Redução da populacão ${ }^{1}$ e infecção de Tibraca limbativentris, após tratamento com isolados (CP) de Beauveria bassiana (Bb) e Metarhizium anisopliae (Ma), em gaiolas no campo.

\begin{tabular}{|c|c|c|c|c|c|}
\hline \multirow[b]{2}{*}{ Tratamento } & \multicolumn{3}{|c|}{$20 \mathrm{DAT}^{2}$} & \multicolumn{2}{|c|}{$90 \mathrm{DAT}^{2}$} \\
\hline & $\begin{array}{c}\text { Insetos } \\
\text { Vivos } \\
\left(\mathrm{N}^{\mathbf{o}}\right)^{4}\end{array}$ & $\begin{array}{l}\text { Eficiênc. } \\
\text { Controle } \\
\qquad(\%)^{3}\end{array}$ & $\begin{array}{l}\text { Insetos } \\
\text { Infect. } \\
\left(\mathrm{N}^{\mathbf{o}}\right)^{4}\end{array}$ & $\begin{array}{c}\text { Insetos } \\
\text { Vivos } \\
\left(\mathrm{N}^{\mathbf{o}}\right)^{4}\end{array}$ & $\begin{array}{l}\text { Eficiênc. } \\
\text { Controle } \\
\qquad \%)^{3}\end{array}$ \\
\hline Testem. & $10,5 \mathrm{a}$ & - & $0 \quad \mathrm{c}$ & 112,6 a & - \\
\hline СРBb164 & $7,2 \mathrm{~b}$ & 32,1 & $2,2 \mathrm{~b}$ & $69,0 \mathrm{~b}$ & 38,7 \\
\hline CPMa122 & $7,8 \mathrm{~b}$ & 26,4 & $1,0 \mathrm{bc}$ & $79,2 \mathrm{~b}$ & 29,7 \\
\hline CPMa 170 & $7,8 \mathrm{~b}$ & 26,4 & $1,6 \mathrm{bc}$ & $85,0 \mathrm{ab}$ & 24,5 \\
\hline CРMa171 & $6,4 \mathrm{~b}$ & 39,6 & $2,2 \mathrm{~b}$ & $67,0 \mathrm{~b}$ & 40,5 \\
\hline СРMa172 & $1,2 \mathrm{c}$ & 88,7 & $5,4 \mathrm{a}$ & $6,4 \mathrm{c}$ & 94,3 \\
\hline
\end{tabular}

${ }^{1}$ Número inicial de insetos/gaiola $=16$ (oito casais).

${ }^{2}$ Dias pós-tratamento com suspensões fúngicas ( $10^{13}$ conídios/ha).

${ }^{3}$ Calculada pela fórmula de Abbott (1925).

${ }^{4}$ Médias com mesma letra não diferem significativamente (Tukey: $\mathrm{P} \leq 0,10$ ). 
Tabela 4. Redução da populacão ${ }^{1}$ e infecção de Tibraca limbativentris, exposto a duas formas de aplicação de isolados (CP) de Beauveria bassiana (Bb) e Metarhizium anisopliae $(M a)$, em gaiolas no campo.

\begin{tabular}{|c|c|c|c|c|c|}
\hline \multirow[b]{2}{*}{ Tratamento $^{2}$} & \multicolumn{3}{|c|}{$20 \mathrm{DAT}^{3}$} & \multicolumn{2}{|c|}{$100 \mathrm{DAT}^{3}$} \\
\hline & $\begin{array}{l}\text { Insetos } \\
\text { Vivos } \\
\left(\mathrm{N}^{\mathrm{o}}\right)^{5}\end{array}$ & $\begin{array}{c}\text { Eficiênc. } \\
\text { Controle } \\
(\%)^{4}\end{array}$ & $\begin{array}{c}\text { Insetos } \\
\text { Infect. } \\
\left(\mathrm{N}^{\mathrm{o}}\right)^{5}\end{array}$ & $\begin{array}{c}\text { Insetos } \\
\text { Vivos } \\
\left(\mathrm{N}^{\mathrm{o}}\right)^{5}\end{array}$ & $\begin{array}{c}\text { Eficiênc. } \\
\text { Controle } \\
(\%)^{4}\end{array}$ \\
\hline Testemunha & $17,1 \mathrm{a}$ & - & $0 \mathrm{c}$ & $31,2 \mathrm{a}$ & - \\
\hline $\mathrm{CPBb} 164(\mathrm{~S})$ & $7,6 \mathrm{~b}$ & 55,5 & $4,8 \mathrm{ab}$ & $10,4 \mathrm{~b}$ & 66,6 \\
\hline $\mathrm{CPBb} 164(\mathrm{G})$ & $9,8 \mathrm{~b}$ & 42,7 & $4,4 a b$ & $15,6 \mathrm{~b}$ & 50,0 \\
\hline CPMa171 (S) & $5,2 \mathrm{~b}$ & 69,6 & $4,2 a b$ & $9,0 \mathrm{~b}$ & 71,1 \\
\hline CPMa171 (G) & $6,1 \mathrm{~b}$ & 64,3 & $5,0 \mathrm{a}$ & $10,8 \mathrm{~b}$ & 65,4 \\
\hline CPMa172 (S) & $5,0 \mathrm{~b}$ & 70,8 & $3,0 \mathrm{abc}$ & $8,6 \mathrm{~b}$ & 72,4 \\
\hline СРMa172 (G) & $8,5 \mathrm{~b}$ & 50,3 & $1,8 \mathrm{bc}$ & $15,2 \mathrm{~b}$ & 51,3 \\
\hline
\end{tabular}

${ }^{1}$ Número inicial de insetos/gaiola $=24$ (doze casais).

${ }^{2}$ Forma de grânulos $(\mathrm{G})$ e de suspensão de conídios $(\mathrm{S})$.

${ }^{3}$ Dias pós-tratamento com suspensões fúngicas $\left(10^{13}\right.$ conídios/ha).

${ }^{4}$ Calculada pela fórmula de Abbott (1925).

${ }^{5}$ Médias com mesma letra não diferem significativamente (Tukey: $\mathrm{P} \leq 0,10$ ).

plantas e principalmente do solo, onde os grânulos concentram-se.

O baixo índice de crescimento de $M$. anisopliae e B. bassiana em T. limbativentris, constatada nos experimentos, pode constituir- se em fator redutor da taxa de disseminação dos fungos nos arrozais e, por conseqüência, dificultar a ocorrência de epizootias. Tal constatação coincide com resultados relacionados às mesmas espécies de fungos,

Tabela 5. Umidade relativa do $\operatorname{ar}(\% \pm \mathrm{EP})^{1}$ durante 20 dias após a aplicação de isolados de Beauveria bassiana e Metarhizium anisopliae sobre Tibraca limbativentris, em gaiolas no campo, no $1^{\circ}, 2^{\circ}$ e $3^{\circ}$ experimentos ${ }^{2}$.

\begin{tabular}{lccc}
\hline \multirow{2}{*}{ Períodos } & \multicolumn{3}{c}{ Umidade Relativa } \\
\cline { 2 - 4 } & $1^{\circ}$ exp. & $2^{\circ}$ exp. & $3^{\circ}$ exp. \\
\hline Até 04 DAT & $75,4 \pm 2,9$ & $80,2 \pm 9,6$ & $76,5 \pm 5,5$ \\
05 a 08 DAT & $75,3 \pm 4,6$ & $80,4 \pm 4,3$ & $79,4 \pm 13,4$ \\
09 a 12 DAT & $65,8 \pm 9,5$ & $91,2 \pm 4,0$ & $80,4 \pm 3,4$ \\
13 a 16 DAT & $82,7 \pm 4,9$ & $76,9 \pm 4,9$ & $83,4 \pm 6,8$ \\
17 a 20 DAT & $76,5 \pm 11,9$ & $82,9 \pm 4,5$ & $85,1 \pm 7,1$ \\
Média geral & $75,2 \pm 8,3$ & $82,2 \pm 7,7$ & $81,0 \pm 8,4$ \\
\hline
\end{tabular}

${ }^{1}$ Valores médios (na atmosfera, fora das gaiolas) para períodos de quatro dias, baseados na média diária de umidade relativa.

${ }^{2}$ Os isolados foram aplicados no $1^{\circ}, 2^{\circ}$ e $3^{\circ}$ experimento, em 14/03/88, 15/02/90 e 22/03/90, respectivamente.

${ }^{3}$ Número de dias pós-aplicação dos tratamentos fúngicos. 
as quais não desenvolviam epizootia em populações naturais de $S$. coarctata (F.) ou mesmo em populações do inseto confinadas em gaiolas (Rombach et al. 1986). A escassez de umidade, foi apontada como a causa principal dos baixos índices de infecção fúngica em $S$. coarctata, em experimentos com gaiolas, indicando que em determinadas circunstâncias nem sempre se estabelecem, entre as plantas de arroz, as condições micrometeorológicas propícias ao desenvolvimento de fungos entomopatogênicos (Carbonell et al. 1980). Portanto, entre as causas do baixo índice de infecção fúngica em T. limbativentris (Tabelas 2, 3 e
5), indicada como favorável à formação de epizootias de ambos os fungos, a campo (Alves 1986). Valores inferiores a 70\% (UR $=65,8 \pm 9,5)$ somente ocorreram entre 9 a 12 DAT, no $1^{\circ}$ experimento. Ademais, o teor de água no solo, no interior das gaiolas, nos três experimentos, foi mantido ao ponto de saturação, possivelmente criando naquele ambiente condições de umidade (do solo e ar) mais elevadas das que ocorreram a nível macrometeorológico.

Provavelmente as condições de temperatura (Tabela 6) tenham afetado o desenvolvimento dos fungos em $T$. limbativentris. Após as aplicações, ocorreram

Tabela 6. Horas $\left(\mathrm{N}^{\mathrm{o}} \pm \mathrm{EP}\right)$ e freqüência $(\%)$ de temperatura ${ }^{1}$ inferior a $22^{\circ} \mathrm{C}$, durante 20 dias após a aplicação (0 a 20 DAT) de isolados de Beauveria bassiana e Metarhizium anisopliae sobre Tibraca limbativentris, em gaiolas no campo, no $1^{\circ}, 2^{\circ}$ e $3^{\circ}$ experimentos ${ }^{2}$.

\begin{tabular}{|c|c|c|c|c|c|c|}
\hline \multirow{2}{*}{ Períodos } & \multicolumn{2}{|c|}{$1^{\circ}$ exp. } & \multicolumn{2}{|c|}{$2^{\circ}$ exp. } & \multicolumn{2}{|c|}{$3^{\circ}$ exp. } \\
\hline & Horas & Freq. & Horas & Freq. & Horas & Freq. \\
\hline Até $04 \mathrm{DAT}^{3}$ & $12,8 \pm 1,5$ & 53,3 & $16,1 \pm 3,3$ & 67,1 & $14,0 \pm 3,8$ & 58,3 \\
\hline 05 a 08 DAT & $11,3 \pm 3,3$ & 46,9 & $9,1 \pm 1,4$ & 38,0 & $20,1 \pm 3,5$ & 83,9 \\
\hline 09 a 12 DAT & $5,8 \pm 1,9$ & 24,0 & $16,8 \pm 6,5$ & 69,8 & $18,8 \pm 3,1$ & 78,1 \\
\hline 13 a 16 DAT & $3,3 \pm 2,9$ & 13,5 & $13,3 \pm 1,9$ & 55,2 & $15,4 \pm 2,4$ & 64,1 \\
\hline 17 a 20 DAT & $19,3 \pm 3,4$ & 80,5 & $14,0 \pm 4,5$ & 58,3 & $5,3 \pm 3,1$ & 21,9 \\
\hline Média geral & $10,4 \pm 5,9$ & 43,3 & $13,9 \pm 4,7$ & 57,8 & $14,7 \pm 6,0$ & 61,1 \\
\hline
\end{tabular}

${ }^{1}$ Valores médios (na atmosfera, fora das gaiolas) para períodos de quatro dias, baseados no número de horas diárias de temperatura abaixo de $22^{\circ} \mathrm{C}$.

${ }^{2}$ Os isolados foram aplicados no $1^{\circ}, 2^{\circ}$ e $3^{\circ}$ experimento, em 14/03/88, 15/02/90 e 22/03/90, respectivamente.

${ }^{3}$ Número de dias pós-aplicação dos tratamentos fúngicos.

4) pode estar incluída a ocorrência de condições ambientais adversas de umidade e temperatura no interior das gaiolas, conforme discutidas por Hall \& Papierok (1982).

Condição adversa de umidade, contudo, não deve ter sido a causa do baixo índice de infecção de T. limbativentris por B. bassiana e $M$. anisopliae. Nos três experimentos, durante 20 DAT, a umidade relativa do ar (UR) permaneceu na faixa de 70 a 100\% (Tabela valores de temperatura aquém de 22 e $24^{\circ} \mathrm{C}$, que representam, respectivamente, o limite inferior da faixa de temperatura considerada ideal para o crescimento e a esporulação de B. bassiana e $M$. anisopliae em insetos, a campo (Alves 1986). Somente abaixo do limite inferior para $B$. bassiana $\left(22^{\circ} \mathrm{C}\right)$, a frequiência de temperatura foi de $43,3 \%$, $57,8 \%$ e $61,1 \%$, no $1^{\circ}, 2^{\circ}$ e $3^{\circ}$ experimento, respectivamente (Tabela 6 ). 
Possivelmente, outros fatores, de natureza biótica, como condições morfo-fisiológicas dos adultos de T. limbativentris, a virulência, a capacidade de reprodução e sobrevivência dos isolados de $B$. bassiana e $M$. anisopliae (Alves 1986) possam, também ter influído no grau variável de mortalidade e de infecção do inseto.

Mesmo evidenciada a necessidade de estudos sobre processos de infecção de ambos os fungos em $T$. limbativentris, os resultados gerais dos três experimentos indicaram que os isolados CPBb164, CPMa171 e CPMa172 são promissores para o controle do inseto.

\section{Agradecimentos}

À Embrapa-CNPAF, pelo fornecimento de fungos e ao Conselho Nacional de Desenvolvimento Científico e Tecnológico (CNPq) pela concessão de Bolsa de Pesquisa, associada ao trabalho.

\section{Literatura Citada}

Abbott, W.S. 1925. A method of computing the effectiveness of an insecticide. J. Econ. Entomol. 18: 265-267.

Alves, S.B. 1986. Controle microbiano de insetos. São Paulo, Manole, 407 p.

Botton, M., J.F. da S. Martins, A. E. Loeck \& M. d'Á. Rosenthal. 1996. Biologia de Tibraca limbativentris Stal s o b r e plantas de arroz. An. Soc. Entomol. Brasil 25: 21-26.

Carbonell, R.M., G.E. Costa \& S.M. Chávez. 1980. Efectividad de Beauveria bassiana (Bals.) Vuill. y Metarhizium anisopliae (Metsch.) Sorok. en el control del Lissorhoptrus brevirostris (Suffr.) (Coleoptera: Curculionidae). Centro Agrícola 7: 107-121.

Costa, E. C. \& D. Link. 1992. Avaliação de danos de Tibraca limbativentris Stal., 1860 (Hemiptera: Pentatomidae) em arroz irrigado. An. Soc. Entomol. Brasil. 21: 187-195.

Ferreira, E., J.F. da S. Martins, P.H.N. Rangel \& V. dos A. Cutrin. 1986. Resistência de arroz ao percevejo-docolmo. Pesq. Agropec. Bras.21: 565-569.

Hall, R.A. \& B. Papierok. 1982. Fungi as biological control agents of arthropods of agricultural and medical importance. Parasitology 84: 205-240.

Martins, J.F. da S., C. Czepack, B.P. Magalhães, E. Ferreira \& J . C . Lord. 1986. Efeito do fungo Metarhizium anisopliae sobre Tibraca limbativentris, percevejo do colmo do arroz. EMBRAPA-CNPAF, Pesq. Andam. 59, $4 \mathrm{p}$.

Martins, J.F. da S. \& M.G.A. de Lima. 1994. Fungos entomopatogênicos no controle do percevejo-do-colmo do arroz Tibraca limbativentris Stal.: Virulência de isolados de Metarhizium anisopliae (Metsch.) Sorok. e Beauveria bassiana (Bals.) Vuill. An. Soc. Entomol. Brasil 23: 39-44.

Marques, E.J., A.M. Villas Boas \& C.E.F. Pereira. 1981. Orientações técnicas para produção do fungo entomógeno Metarhizium anisopliae (Metsch.) em laboratórios setoriais. P i r a c i c a b a, PLANALSUCAR, Boletim Técnico 2, 23p.

Rombach, M.C., R.M. Aguda, B.M. Sphepard \& D.W. Roberts. 1986. Entomopathogenic fungi in the control of the black bug of rice, Scotinophara coarctata (Hemiptera: Pentatomidae). J. Invertebr. Pathol. 48: 174-179.

SAS Institute. 1985. SAS user's guide: statistics, version 5 edition. Cary, NC. SAS Institute Inc. 956p.

Recebido em 10/01/96. Aceito em 23/06/97. 